\title{
Computational limits to nonparametric estimation for ergodic processes
}

\author{
Hayato Takahashi, Member, IEEE
}

\begin{abstract}
A new negative result for nonparametric distribution estimation of binary ergodic processes is shown. The problem of estimation of distribution with any degree of accuracy is studied. Then it is shown that for any countable class of estimators there is a zero-entropy binary ergodic process that is inconsistent with the class of estimators. Our result is different from other negative results for universal forecasting scheme of ergodic processes.
\end{abstract}

Index Terms-ergodic process, cutting and stacking, nonparametric estimation, computable function.

\section{INTRODUCTION}

Let $X_{1}, X_{2}, \ldots$ be a binary-valued ergodic process and $P$ be its distribution. In this paper we study nonparametric estimation of binary-valued ergodic processes with any degree of accuracy. Let $S$ and $\Omega$ be the set of finite binary strings and the set of infinite binary sequences, respectively. Let $\Delta(x):=\{x \omega \mid \omega \in \Omega\}$, where $x \omega$ is the concatenation of $x \in S$ and $\omega$, and write $P(x)=P(\Delta(x))$. For $x \in S,|x|$ is the length of $x$. Let $\mathbb{N}, \mathbb{Z}$, and $\mathbb{Q}$ be the set of natural numbers, the set of integers, and the set of rational numbers, respectively. From ergodic theorem, there is a function $r$ such that for $x \in S, n, k \in \mathbb{N}$,

$$
\begin{gathered}
P\left(\cup \left\{\Delta(y)|| P(x)-\frac{1}{|y|} \sum_{i=1}^{|y|-|x|+1} I_{y_{i}^{i+|x|-1}=x} \mid \geq 1 / k,\right.\right. \\
|y|=n\})<r(n, k, x), \\
\forall x, k \lim _{n} r(n, k, x)=0,
\end{gathered}
$$

where $I$ is the indicator function and $y_{i}^{j}=y_{i} y_{i+1} \cdots y_{j}$ for $y=y_{1} \cdots y_{n}, i \leq j \leq n . r$ is called convergence rate. If $r$ is given, we know how much sample size is necessary to estimate the distribution with prescribed accuracy. However it is known that there is no universal convergence rate for ergodic theorem. If $r$ is not known, ergodic theorem does not help to estimate the distribution with prescribed accuracy. Here a natural question arise: for any binary-valued ergodic process, is it always possible to estimate the distribution with any degree of accuracy with positive probability? We show that this problem has a negative answer, i.e., for any countable class of estimators there is a zero-entropy binary ergodic process that is not estimated from this class of estimators with positive probability. In particular, since the set of computable functions is countable, we see that there is a zero-entropy binary ergodic process that is inconsistent with computable estimators. Our

The author is with the Institute of Statistical Mathematics, 10-3 Midori-cho, Tachikawa, Tokyo 190-8562, Japan. e-mail: hayato.takahashi@ieee.org. result is not derived from other negative results for universal forecasting scheme of ergodic processes, see Remark 1

Let $x \sqsubseteq y$ if $x$ is a prefix of $y . f$ is called estimator if $\exists D_{f} \subseteq S \times \mathbb{N} \times S f: D_{f} \rightarrow \mathbb{Q}$ and

$$
\begin{aligned}
& f(x, k, y) \text { is defined, i.e., }(x, k, y) \in D_{f} \\
& \Rightarrow \forall z \sqsupseteq y f(x, k, z)=f(x, k, y) .
\end{aligned}
$$

For $\omega \in \Omega$, let $f(x, k, \omega):=f(x, k, y)$ if $f(x, k, y)$ is defined and $y \sqsubset \omega$. We say that $f$ estimates $P$ if

$$
\begin{aligned}
P(\omega \mid \forall x, k & f(x, k, \omega) \text { is defined and } \\
& \left.|P(x)-f(x, k, \omega)|<\frac{1}{k}\right)>0 .
\end{aligned}
$$

Here $\omega$ is a sample sequence and the minimum length of $y \sqsubset \omega$ for which $f(x, k, y)$ is defined is a stopping time.

In this paper, we construct an ergodic process that is not estimated from any given countable set of estimators:

\section{Theorem 1.}

$$
\forall F \text { : countable set of estimators }
$$

$\exists P$ ergodic and zero entropy $\forall f \in F$ $P(\omega \mid \forall x, k f(x, k, \omega)$ is defined and

$$
\left.|P(x)-f(x, k, \omega)|<\frac{1}{k}\right)=0 .
$$

We say that $P$ is effectively estimated if there is a partial computable $f$ that satisfies (2) and (3). Since the set of partial computable estimators is countable, we have

Corollary 1. There is a zero entropy ergodic process that is not effectively estimated.

If $r$ in (1) is computable then it is easy to see that $P$ is effectively estimated 11 For example, i.i.d. processes of finite alphabet are effectively estimated, see Leeuw et al. [3].

As stated above, a difficulty of effective estimation of ergodic processes comes from that there is no universal convergence rate for ergodic theorem. In Shields pp.171 [7], it is shown that for any given decreasing function $r$, there is an ergodic process that satisfies

$$
\exists N \forall n \geq N P\left(\left|P(1)-\sum_{i=1}^{n} I_{X_{i}=1} / n\right| \geq 1 / 2\right)>r(n) .
$$

In particular if $r$ is chosen such that $r$ decreases to 0 asymptotically slower than any computable function then $r$ is not

\footnotetext{
${ }^{1}$ More precisely if $r$ is upper semi-computable (approximated from above by some algorithm), $P$ is effectively estimated.
} 
computable. In V'yugin [8], a binary-valued computable stationary process with incomputable convergence rate is shown.

It is possible that an ergodic process is effectively estimated even if the convergence rate is not computable (nor upper semi-computable).

Theorem 2. For any decreasing $r$, there is a zero entropy ergodic process that is effectively estimated and satisfies (4).

Remark 1. In Cover [2], two problems about prediction of ergodic processes are posed. Problem 1 : Is there a universal scheme $f$ such that $\lim _{n \rightarrow \infty}\left|f\left(X_{0}^{n-1}\right)-P\left(X_{n} \mid X_{0}^{n-1}\right)\right| \rightarrow$ 0 , a.s. for all binary-valued ergodic $P$ ? Problem 2 : Is there a universal scheme $f$ such that $\lim _{n \rightarrow \infty} \mid f\left(X_{n}^{-1}\right)-$ $P\left(X_{0} \mid X_{-\infty}^{-1}\right) \mid \rightarrow 0$, a.s. for all binary-valued ergodic $P$ ? Problem 2 was affirmatively solved by Ornstein [5], [9]. Problem 1 has a negative answer as follows (Bailey, Ryabko, see [1], [6], [4]): For any $f$ there is a binary-valued ergodic process $X_{1}, X_{2}, \ldots$ such that

$$
P\left(\limsup _{n \rightarrow \infty}\left|f\left(X_{0}^{n-1}\right)-P\left(X_{n} \mid X_{0}^{n-1}\right)\right|>0\right)>0 .
$$

It is not difficult to see that the above result is extended to a countable class $\left\{f_{1}, f_{2}, \ldots\right\}$, i.e., for any $\left\{f_{1}, f_{2}, \ldots\right\}$ there is an ergodic process such that (5) holds for all $f_{1}, f_{2}, \ldots$. However this result does not imply Theorem 1 In fact, there is a finite-valued ergodic process that is effectively estimated but satisfies (5), see below. Roughly speaking, one of the difference between these problems is that in Problem 1 we have to estimate $P\left(X_{n} \mid X_{0}^{n-1}\right)$ from $X_{0}^{n-1}$, however in our estimation scheme, sample size is a stopping time and we can use a sufficiently large sample $X_{0}^{m}, m>n$ to estimate $P\left(X_{0}^{n}\right)$.

In Ryabko [6], the process for (5) is constructed as follows: First consider an ergodic Markov process $Y_{1}, Y_{2}, \ldots$ on a countable state $0,1,2, \ldots$ and $P_{i, i+1}:=1 / 2, P_{i, 0}:=1 / 2$ for $i=0,1, \ldots$, where $P_{i, j}$ is the transition probability from $i$ to $j$. The process $X_{i} \in\{0,1,2\}, i \in \mathbb{N}$ is defined by $X_{i}=0$ if $Y_{i}=0$ and $P\left(X_{i}=1\right)=p_{j}, P\left(X_{i}=2\right)=1-p_{j}$ if $Y_{i}=j \geq 1$. Then $\left\{X_{i}\right\}$ is ergodic. In particular, for any $\left\{f_{1}, f_{2}, \ldots\right\}$, we can choose $\left\{p_{1}, p_{2}, \ldots\right\}$ such that (5) holds for all $f_{i}, i \in \mathbb{N}$. However $\left\{X_{i}\right\}$ is effectively estimated for any $\left\{p_{i}\right\}$ as follows. Let

$$
I_{j}=\left\{i \mid X_{i}=0 \text { and } X_{k} \neq 0 \text { for } i<k \leq i+j\right\} .
$$

From the construction, we have

$$
X_{i}=0 \text { and } X_{k} \neq 0 \text { for } i<k \leq i+j \Leftrightarrow Y_{i+j}=j .
$$

Since the above event has a positive probability, $I_{j}$ is an infinite set with probability one. Since $Y_{i+j}=j$ for $i \in I_{j}$, $\left\{X_{i+j}\right\}_{i \in I_{j}}$ are i.i.d. random variables with $P\left(X_{i+j}=1\right)=$ $p_{j}, P\left(X_{i+j}=2\right)=1-p_{j}$. Thus we can estimate $p_{j}$ with any degree of accuracy for all $j$. Since the process $\left\{X_{i}\right\}$ is determined from $\left\{p_{i}\right\}$, it is effectively estimated.

\section{Cutting And Stacking}

We construct ergodic processes in Theorem 1 and 2 by cutting and stacking method. The basic idea of our constructions are similar to that of (4). In this section, we briefly introduce some notions about cutting and stacking, which we need in the proof.

Let $X:=[0,1]$ and consider Lebesgue measure $\lambda$ on $(X, \mathcal{B})$, where $\mathcal{B}$ is the Borel $\sigma$-field. We construct an ergodic transformation $T$ on $(X, \mathcal{B}, \lambda)$. Let $\mathcal{C}:=\left(L_{1}, L_{2}, \ldots, L_{n}\right)$ be an ordered set of mutually disjoint intervals of equal length. $\mathcal{C}$ is called column. $w(\mathcal{C}):=\lambda\left(L_{1}\right), h(\mathcal{C}):=n$, and $S(\mathcal{C}):=\cup_{i} L_{i}$ are called width, height, and support of $\mathcal{C}$, respectively. Two columns are called disjoint if their support are disjoint. For two disjoint columns $\mathcal{C}:=\left(L_{1}, L_{2}, \ldots, L_{n}\right)$ and $\mathcal{C}^{\prime}:=\left(L_{1}^{\prime}, L_{2}^{\prime}, \ldots, L_{m}^{\prime}\right)$ of the same width, let $\mathcal{C} *$ $\mathcal{C}^{\prime}:=\left(L_{1}, \ldots, L_{n}, L_{1}^{\prime}, \ldots, L_{m}^{\prime}\right)$. For a given column $\mathcal{C}=$ $\left\{L_{i}\right\}_{1 \leq i \leq n}$, two disjoint columns $\mathcal{C}_{L}:=\left\{L_{i}^{1}\right\}_{1 \leq i \leq n}$ and $\mathcal{C}_{R}:=\left\{L_{i}^{2}\right\}_{1 \leq i \leq n}$ are called partition of $\mathcal{C}$ if $L_{i}=L_{i}^{1} \cup L_{i}^{2}$ and $L_{i}^{1} \cap L_{i}^{2}=\emptyset$ for $1 \leq i \leq n$ and $w\left(\mathcal{C}_{R}\right)=w\left(\mathcal{C}_{L}\right)=\frac{1}{2} w(\mathcal{C})$. In order to specify the partition, we require that the leftendpoint of $L_{i}^{1}$ is less than that of $L_{i}^{2}$. Let $\mathcal{C} * \mathcal{C}:=$ $\mathcal{C}_{L} * \mathcal{C}_{R}$, where $\mathcal{C}_{L}$ and $\mathcal{C}_{R}$ are partition of $\mathcal{C}$, see Fig. 1 Let $\mathcal{C}(0):=\mathcal{C}$ and $\mathcal{C}(n+1):=\mathcal{C}(n) * \mathcal{C}(n)$ for $n \geq 0$. We have $w(\mathcal{C}(n+1))=\frac{1}{2} w(\mathcal{C}(n))=2^{-(n+1)} w(\mathcal{C})$ and $h(\mathcal{C}(n+1))=2 h(\mathcal{C}(n))=2^{n+1} h(\mathcal{C})$.

A transformation $T$ is defined on a column $\mathcal{C}:=$ $\left(L_{1}, \ldots, L_{n}\right)$ by 1) $T\left(L_{i}\right)=L_{i+1}$ and $T\left(a_{i}+y\right)=a_{i+1}+y$, where $0 \leq y \leq w(\mathcal{C})$, and $a_{i}$ is the left-endpoint of $L_{i}$ for $1 \leq i \leq n-1$, and 2) $T$ is not defined on $L_{n}$. Then $T$ is a measure preserving transformation defined on intervals of $\mathcal{C}$ except for $L_{n}$. Similarly, $T^{-1}$ is defined on $\mathcal{C}$ except for $L_{1}$. Note that $T$ (and $T^{-1}$ ) defined by $\mathcal{C} * \mathcal{C}$ extends $T$ (and $T^{-1}$ ) defined by $\mathcal{C}$, respectively.

We say that a sequence of columns $\mathcal{C}_{1}, \mathcal{C}_{2}, \ldots$ is extending if $S\left(\mathcal{C}_{n}\right) \subseteq S\left(\mathcal{C}_{n+1}\right)$ and $T$ defined by $\mathcal{C}_{n+1}$ extends $T$ defined by $\mathcal{C}_{n}$ for all $n$. Suppose that there is an extending sequence of columns $\mathcal{C}_{1}, \mathcal{C}_{2}, \ldots$ such that $\lim _{n} w\left(\mathcal{C}_{n}\right)=0$ and $\lambda\left(\cup_{n} S\left(\mathcal{C}_{n}\right)\right)=1$. Then we see that an invertible measurepreserving transformation $T: X \rightarrow X$ is uniquely defined except for a null set. $T$ is ergodic as follows: Suppose that $A \subseteq X$ is a nontrivial invariant set, i.e., $T(A)=A$ and $0<\lambda(A)<1$. Since $\lim _{n} w\left(\mathcal{C}_{n}\right)=0$ and $\lambda\left(\cup_{n} S\left(\mathcal{C}_{n}\right)\right)=1$, from Lebesgue density theorem, there are $n$ and $L_{i}, L_{j} \in \mathcal{C}_{n}$ such that $1 / 2<\lambda\left(A \cap L_{i}\right) / \lambda\left(L_{i}\right), 1 / 2<\lambda\left(A^{c} \cap L_{j}\right) / \lambda\left(L_{j}\right)$. Then $\lambda\left(A \cap A^{c}\right)=\lambda\left(T^{j-i}(A) \cap A^{c}\right) \geq \lambda\left(T^{j-i}\left(A \cap L_{i}\right) \cap\right.$ $\left.A^{c} \cap L_{j}\right)>0$, which is a contradiction.

Let $X^{0}$ and $X^{1}$ be measurable sets of $X$ such that $X^{0} \cup X^{1}=X$ and $X^{0} \cap X^{1}=\emptyset$. For $\xi \in X$, let $\phi(\xi)=\cdots \xi(-1) \xi(0) \xi(1) \cdots \in\{0,1\}^{\mathbb{Z}}$, where $\xi(i)=0$ if $T^{i}(\xi) \in X^{0}$ and 1 else for all $i \in \mathbb{Z}$. Let $P:=\lambda \circ \phi^{-1}$. If $T$ is an invertible ergodic transformation, $P$ is an invertible ergodic process on $\{0,1\}^{\mathbb{Z}}$ and is called $\left(T, X^{0}, X^{1}\right)$ process. We say that a column $\left(L_{1}, \ldots, L_{n}\right)$ is compatible with $\left(X^{0}, X^{1}\right)$ if $\forall 1 \leq i \leq n, L_{i} \subseteq X^{0}$ or $L_{i} \subseteq X^{1}$, and in that case let $s\left(L_{i}\right):=0$ if $L_{i} \subseteq X^{0}$ and 1 else for $1 \leq i \leq n$, and $s\left(L_{1}, \ldots, L_{n}\right):=s\left(L_{1}\right) \cdots s\left(L_{n}\right)$. 


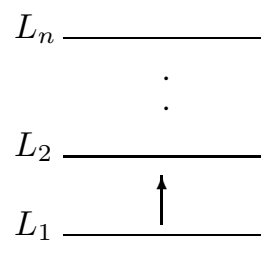

(a) $\mathcal{C}=\left(L_{1}, \ldots, L_{n}\right)$

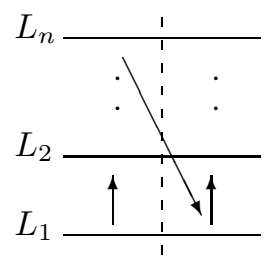

(b) $\mathcal{C} * \mathcal{C}$
Fig. 1. Cutting and stacking

\section{Proof of Theorem 1}

Let $F:=\left\{f_{1}, f_{2}, \ldots\right\}$ be a countable set of estimators. Consider the following three statements:

$$
\begin{aligned}
& \forall P \text { ergodic on } \Omega \exists f \in F \\
& P\left(\omega|\forall x, k| P(x)-f(x, k, \omega) \mid<\frac{1}{k}\right)>0,
\end{aligned}
$$

$\forall P$ ergodic on $\Omega \exists f \in F$

$$
P\left(\omega|\forall m, k| P\left(0^{m}\right)-f\left(0^{m}, k, \omega\right) \mid<\frac{1}{k}\right)>0,
$$

$\forall P$ ergodic on $\Omega \exists \hat{f} \in \hat{F} P(\omega \mid \hat{f}(\omega)=R)>0$,

where $0^{m}$ is the $m$-times concatenation of 0 's,

$$
\begin{gathered}
R:=\left\{(n, m) \mid P\left(0^{m}\right)<2^{-(n+2)}\right\}, \\
\hat{f}:=\left\{(n, m, y) \mid \exists k f\left(0^{m}, k, y\right)+\frac{1}{k}<2^{-(n+2)}\right\}, \\
\hat{F}:=\{\hat{f} \mid f \in F\}, \\
\hat{f}(x):=\{(n, m) \mid(n, m, y) \in \hat{f}, y \sqsubseteq x\}, \hat{f}(\omega):=\cup_{x \sqsubset \omega} \hat{f}(x) .
\end{gathered}
$$

Then we have $(6) \Rightarrow(7) \Rightarrow(8)$, where $(7) \Rightarrow(8)$ follows from

$$
\forall m, k\left|P\left(0^{m}\right)-f\left(0^{m}, k, \omega\right)\right|<\frac{1}{k} \Rightarrow \hat{f}(\omega)=R .
$$

Therefore in order to show Theorem 1 , it is sufficient to negate (8) (Lemma 1).

Lemma 1. For any countable $F$, there is a zero entropy ergodic $P$ such that

$\forall \hat{f} \in \hat{F}, P(\omega \mid \hat{f}(\omega)=R)=0$.

Proof) Let $\hat{F}:=\left\{\hat{f}_{1}, \hat{f}_{2}, \ldots\right\}$. We construct an ergodic process inductively by cutting and stacking method such that if there are $a, x, e$ such that $a \in \hat{f}_{e}(x)$ at some stage then the process is made to falsify $\hat{f}_{e}$, i.e., $a \notin R$.

Let $X^{0}:=[0,1 / 2)$ and $X^{1}:=[1 / 2,1]$. For $n \geq 1$, let $A_{n}:=\left(2^{-(n+1)}, 2^{-n}\right]$. We construct inductively an extending sequence of columns $\mathcal{C}_{0}, \mathcal{C}_{1}, \ldots$, which are compatible with $\left(X^{0}, X^{1}\right), \lim _{n} w\left(\mathcal{C}_{n}\right)=0$, and $\cup_{n} S\left(\mathcal{C}_{n}\right)=\cup_{i \in J} A_{i} \cup X^{1}$, where $J$ is defined simultaneously with columns.

Stage 0 : Let $\mathcal{C}_{0}:=X^{1}, G_{0}:=\emptyset$, and $k_{0}:=1$.

Stage $n$ : Suppose that $G_{n-1}$ is defined and $\mathcal{C}_{0}, \ldots, \mathcal{C}_{n-1}$ are extending and compatible with $\left(X^{0}, X^{1}\right)$. Let $\mathcal{C}_{n-1}:=$ $\left(L_{1}, \ldots, L_{h_{n-1}}\right)$ and suppose that $w\left(\mathcal{C}_{n-1}\right)=2^{-k_{n-1}}$ for $k_{n-1} \in \mathbb{N}$. Let

$$
\begin{aligned}
F_{n}:=\{(\langle e, i\rangle, m) & \mid 1 \leq e \leq n, 1 \leq i \leq h_{n-1} \\
& \left.(\langle e, i\rangle, m) \in \hat{f}_{e}\left(s\left(L_{i} \cdots L_{h_{n-1}}\right)\right)\right\}
\end{aligned}
$$

$$
G_{n}:=\left\{\langle e, i\rangle \mid \exists m(\langle e, i\rangle, m) \in F_{n}\right\} \cap\left(G_{n-1}\right)^{c},
$$

where $\langle\cdot, \cdot\rangle: \mathbb{N} \times \mathbb{N} \rightarrow \mathbb{N}$ is a bijection and $\left(G_{n-1}\right)^{c}$ is the complement of $G_{n-1}$.

If $G_{n}=\emptyset$ then set $k_{n}:=k_{n-1}+1$ and $\mathcal{C}_{n}:=\mathcal{C}_{n-1}(1)$.

If $G_{n} \neq \emptyset$ then let

$$
\begin{aligned}
& m(e, i):=\min \left\{m \mid(\langle e, i\rangle, m) \in F_{n}\right\} \text { for }\langle e, i\rangle \in G_{n}, \text { and } \\
& k_{n}:= \\
& \quad \max \left\{k_{n-1}+1,\right. \\
& \left.\quad \min \left\{t \in \mathbb{N} \mid \forall\langle e, i\rangle \in G_{n}, 2^{t-\langle e, i\rangle-1} \geq 2 m(e, i)\right\}\right\} .
\end{aligned}
$$

Since $w\left(\mathcal{C}_{n-1}\right)=2^{-k_{n-1}}$ and $w\left(A_{\langle e, i\rangle}\right)=2^{-(\langle e, i\rangle+1)}$, we have

$$
w\left(\mathcal{C}_{n-1}\left(k_{n}-k_{n-1}\right)\right)=w\left(A_{\langle e, i\rangle}\left(k_{n}-\langle e, i\rangle-1\right)\right)=2^{-k_{n}} .
$$

Define

$\mathcal{C}_{n}:=\mathcal{C}_{n-1}\left(k_{n}-k_{n-1}\right) * A_{n_{1}}\left(k_{n}-n_{1}-1\right) * \cdots * A_{n_{t}}\left(k_{n}-n_{t}-1\right)$,

where $G_{n}=\left\{n_{1}<n_{2}<\cdots<n_{t}\right\}$.

By induction, we have constructed an extending sequence of columns $\mathcal{C}_{0}, \mathcal{C}_{1}, \ldots$, which are compatible with $\left(X^{0}, X^{1}\right)$ and $w\left(\mathcal{C}_{n}\right)=2^{-k_{n}}$. Let $J:=\cup_{n} G_{n}$ then $\cup_{n} S\left(\mathcal{C}_{n}\right)=\cup_{i \in J} A_{i} \cup$ $X^{1}$.

Let $\Omega_{1}:=\cup_{n} S\left(\mathcal{C}_{n}\right)$. Let $T: \Omega_{1} \rightarrow \Omega_{1}$ be an invertible measure preserving transformation defined by $\cup_{n} \mathcal{C}_{n}$ and $P$ be the $\left(T, X^{0}, X^{1}\right)$ process, then $P$ is ergodic.

Let

$$
R:=\left\{(n, m) \mid P\left(0^{m}\right)<2^{-(n+2)}\right\} .
$$

Suppose that there is an $e$ such that

$$
P\left(\omega \mid \hat{f}_{e}(\omega)=R\right)>0 .
$$

Since $k_{n-1}<k_{n}$ for all $n$, we have $\lim _{n} h\left(\mathcal{C}_{n}\right)=\infty$. Since $\forall n, s\left(\mathcal{C}_{n-1}\right) \sqsubseteq s\left(\mathcal{C}_{n}\right)$, we see that $s\left(\mathcal{C}_{1}\right), s\left(\mathcal{C}_{2}\right), \ldots$ defines a unique sequence $\alpha:=\alpha_{1} \alpha_{2} \cdots \in \Omega, \forall i, \alpha_{i} \in\{0,1\}$ in the limit, i.e., $\forall n, s\left(\mathcal{C}_{n}\right) \sqsubset \alpha$. Since $\Omega_{1}=\cup_{n} S\left(\mathcal{C}_{n}\right)$, we have

$$
\begin{aligned}
& \xi \in \Omega_{1} \Leftrightarrow \exists n, i, 1 \leq i \leq h_{n}, \xi \in L_{i}, \mathcal{C}_{n}=\left(L_{1}, \ldots, L_{h_{n}}\right), \\
& \exists i \xi(0) \cdots \xi\left(h_{n}-i\right)=\alpha_{i} \cdots \alpha_{h_{n}},
\end{aligned}
$$

where $h_{n}=h\left(\mathcal{C}_{n}\right)$. From (9), (13), and (14), there are $i, n \in$ $\mathbb{N}, 1 \leq i \leq h_{n-1}$ such that

$$
\hat{f}_{e}\left(\alpha_{i} \cdots \alpha_{h_{n-1}}\right) \subseteq R \text { and }\langle e, i\rangle \in G_{n} .
$$

From (10), we have

$$
s\left(A_{\langle e, i\rangle}\left(k_{n}-\langle e, i\rangle-1\right)\right)=0^{2^{k_{n}-\langle e, i\rangle-1}} \sqsupseteq 0^{2 m(e, i)} .
$$

Let $\left(L_{1}, \ldots, L_{h}\right):=A_{\langle e, i\rangle}\left(k_{n}-\langle e, i\rangle-1\right)$. If $\xi \in \cup_{1 \leq j \leq h / 2} L_{j}$ then $\phi(\xi) \sqsupseteq 0^{m(e, i)}$. Since $\lambda\left(S\left(A_{\langle e, i\rangle}\right)\right)=2^{-(\langle e, i\rangle+1)}$, we have

$$
P\left(0^{m(e, i)}\right) \geq \lambda\left(\cup_{1 \leq j \leq h / 2} L_{j}\right)=2^{-(\langle e, i\rangle+2)} .
$$

Then $(\langle e, i\rangle, m(e, i)) \in \hat{f}_{e}\left(\alpha_{i} \alpha_{i+1} \cdots\right)$ and $(\langle e, i\rangle, m(e, i)) \notin$ $R$, which contradicts to (15), see Fig. 2. Thus we have $\forall e, P\left(\omega \mid \hat{f}_{e}(\omega)=R\right)=0$. 
Next we show that the entropy is zero. From (11), (12), and (14), for $1 \leq i \leq j \leq h_{n}$, we have $P\left(\alpha_{i} \cdots \alpha_{j}\right) \geq$ $\lambda\left(w\left(\mathcal{C}_{n}\right)\right)=2^{-k_{n}}$ and $h_{n} \geq 2^{k_{n}-k_{n-1}} h_{n-1} \geq 2^{k_{n}-k_{0}} h_{0}=$ $2^{k_{n}-1}$. Since $1 / 2 \leq \lambda\left(S\left(\mathcal{C}_{n}\right)\right)$, we have $\frac{1}{4} \leq \lambda\left(\cup_{1 \leq i \leq h_{n} / 2} L_{i}\right)$ and

$$
\forall n P\left(\frac{-\log _{2} P\left(\omega_{1} \cdots \omega_{h_{n} / 2}\right)}{h_{n} / 2} \leq k_{n} 2^{-k_{n}+2}\right) \geq 1 / 4 .
$$

Suppose that the entropy of $P$ is positive. Since $\lim _{n} k_{n}=\infty$ and $\lim _{n} h_{n}=\infty$, from Shannon-McMillan-Breiman theorem,

$\forall \epsilon \exists N \forall n \geq N P\left(-\log _{2} P\left(\omega_{1} \cdots \omega_{n}\right) / n>k_{n} 2^{-k_{n}+2}\right)>1-\epsilon$, which contradicts to (16), and the entropy of $P$ is zero.

$$
\begin{gathered}
s\left(\mathcal{C}_{n-1}\right)=\alpha_{1} \cdots \alpha_{i} \cdots \alpha_{h}, \hat{f}_{e}\left(\alpha_{i} \cdots \alpha_{h}\right) \ni(\langle e, i\rangle, m) \\
\Downarrow \\
s\left(\mathcal{C}_{n}\right)=\overbrace{\alpha_{1} \cdots \alpha_{h}}^{\Downarrow\left(\mathcal{C}_{n-1}\right)} \cdots \overbrace{\alpha_{1} \cdots \alpha_{h}}^{s\left(\mathcal{C}_{n-1}\right)} 0 \cdot \overbrace{00}^{\exists 0_{0}^{m}} .
\end{gathered}
$$

Fig. 2. Example of construction. For simplicity, suppose that $s\left(\mathcal{C}_{n-1}\right)=$ $\alpha_{1} \cdots \alpha_{h}, G_{n}=\{\langle e, i\rangle\}$, and $(\langle e, i\rangle, m) \in \hat{f}_{e}\left(\alpha_{i} \cdots \alpha_{h}\right)$. Then by stacking a long column of $A_{\langle e, i\rangle}, \hat{f}_{e}\left(\alpha_{i} \cdots \alpha_{h}\right)$ fails to guess $R$. We choose $a, b$ such that $w\left(\mathcal{C}_{n-1}(a)\right)=w\left(A_{\langle e, i\rangle}(b)\right), 2^{b} \geq 2 m$ and let $\mathcal{C}_{n}:=\mathcal{C}_{n-1}(a) * A_{\langle e, i\rangle}(b)$. Then by considering the trajectories starting from the first half levels of $A_{\langle e, i\rangle}(b)$, we have $P\left(0^{m}\right) \geq \lambda\left(S\left(A_{\langle e, i\rangle}\right)\right) / 2=$

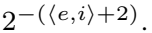

\section{Proof of TheOREM 2}

\section{A. Construction of a process for (4)}

Here we summarize the construction of the process for (4), which we use in Theorem 2 (Actually independent cutting and stacking method is used in [7], however we need not it here.)

We construct an extending sequence of columns $\mathcal{C}_{n}, n=$ $0,1,2, \ldots$ from $k_{0}=1<k_{1}<k_{2}<\cdots \in \mathbb{N}$ by induction. Let $X:=[0,1], X^{0}=[0,1 / 2], X^{1}=(1 / 2,1], \mathcal{C}_{0}:=X^{1}$, and $A_{n}:=\left(2^{-(n+1)}, 2^{-n}\right]$ for $n=1,2, \ldots$.

Stage $n$ : Suppose that $\mathcal{C}_{n-1}$ is defined and $w\left(\mathcal{C}_{n-1}\right)=$ $2^{-k_{n-1}}$. Since $w\left(\mathcal{C}_{n-1}\left(k_{n}-k_{n-1}\right)\right)=w\left(A_{n}\left(k_{n}-(n+1)\right)\right)=$ $2^{-k_{n}}$, define

$$
\mathcal{C}_{n}:=\mathcal{C}_{n-1}\left(k_{n}-k_{n-1}\right) * A_{n}\left(k_{n}-(n+1)\right) .
$$

Then

$$
\begin{aligned}
& w\left(\mathcal{C}_{n}\right)=2^{-k_{n}}, \\
& S\left(\mathcal{C}_{n}\right)=\cup_{i=1}^{n} A_{i} \cup X^{1} \text { and } \lambda\left(S\left(\mathcal{C}_{n}\right)\right)=1-2^{-(n+1)}, \\
& h\left(\mathcal{C}_{n}\right)=\lambda\left(S\left(\mathcal{C}_{n}\right)\right) / w\left(\mathcal{C}_{n}\right)=2^{k_{n}}\left(1-2^{-(n+1)}\right) .
\end{aligned}
$$

Since $\lim _{n} w\left(\mathcal{C}_{n}\right)=0$ and $\lambda\left(\cup_{n} \mathcal{C}_{n}\right)=1, \cup_{n} \mathcal{C}_{n}$ defines an invertible ergodic process $T$. Let $P$ be the $\left(T, X^{0}, X^{1}\right)$ process. Let $\left.A_{n}\left(k_{n}-(n+1)\right)\right)=\left(L_{1}, \ldots, L_{h}\right), h=2^{k_{n}-(n+1)}$. Since $A_{n} \subseteq X^{0}$, we have $s\left(L_{1}, \ldots, L_{h}\right)=0^{h}$ and if $\xi \in \cup_{i=1}^{h^{\prime}} L_{i}$ then $\xi(0) \xi(1) \cdots \xi\left(h^{\prime}-1\right)=0^{h^{\prime}}$ for $h^{\prime}=h / 2$. Since $\lambda\left(\cup_{i=1}^{h^{\prime}} L_{i}\right)=2^{-(n+2)}$, we have

$$
2^{-(n+2)}<P\left(0^{h^{\prime}}\right) \leq P\left(\left|P(0)-\sum_{i=1}^{h^{\prime}} I_{X_{i}=0} / h^{\prime}\right| \geq 1 / 2\right) .
$$

Thus by choosing $\left\{k_{i}\right\}$, we can construct an ergodic process with arbitrary slow convergence rate.

\section{B. Proof}

We show Theorem 2 for the ergodic process defined in Section IV-A

In the following we write $x^{n}$ as the $n$-times concatenation of $x \in S$, e.g., $(01)^{2}=0101$. From (17), we have

$$
s\left(\mathcal{C}_{n}\right)=\left(s\left(\mathcal{C}_{n-1}\right)\right)^{2^{k_{n}-k_{n-1}}} 0^{2^{k_{n}-(n+1)}} .
$$

For example, if $k_{0}=1, k_{1}=2, k_{2}=3$ then

$$
s\left(\mathcal{C}_{0}\right)=1, s\left(\mathcal{C}_{1}\right)=110, s\left(\mathcal{C}_{2}\right)=1101100 .
$$

From (17), (18), and (19), we see that

$$
\begin{aligned}
& P\left(01^{2^{k_{1}-1}} 0\right)=w\left(\mathcal{C}_{1}\right)=2^{-k_{1}}, \\
& P\left(01^{n} 0\right)=0 \text { if } n \neq 2^{k_{1}-1} \text { and } n \neq 0, \\
& P\left(10^{f(n)} 1\right)=w\left(\mathcal{C}_{n}\right)=2^{-k_{n}}, f(n)=\sum_{i=1}^{n} 2^{k_{i}-(i+1)}, \\
& P\left(10^{m} 1\right)=0 \text { if } \forall n m \neq f(n) \text { and } m \neq 0 .
\end{aligned}
$$

Let

$$
B_{0}:=\mathcal{C}_{0} \text { and } B_{n}:=\cup_{i=1}^{\left(2^{k_{n}-k_{n-1}}-1\right) h\left(\mathcal{C}_{n-1}\right)} L_{i},
$$

for $\mathcal{C}_{n}=\left(L_{1}, \ldots, L_{h_{n}}\right), n \geq 1$. From (17) and Lemma 2 below, we have

$$
\lambda\left(\cap_{i=0}^{n} B_{i}\right)=\lambda\left(B_{0}\right) \Pi_{i=1}^{n}\left(1-2^{-\left(k_{i}-k_{i-1}\right)}\right),
$$

see Fig. 3 Assume that $\forall i k_{i}-k_{i-1} \geq i$. Since $\sum_{i=1}^{\infty} 2^{-\left(k_{i}-k_{i-1}\right)} \leq 1$, we have

$$
\lambda\left(\cap_{i=0}^{\infty} B_{i}\right)>0 .
$$

Let $\xi \in \cap_{i=0}^{\infty} B_{i}$ and $\phi(\xi)^{\prime}:=\xi(0) \xi(1) \cdots \in \Omega$. Then

$(*)$ the first time that the pattern $10^{n} 1$ appears in $\phi(\xi)^{\prime}$ is less than that of $10^{m} 1$ if $n<m, P\left(10^{n} 1\right)>0$, and $P\left(10^{m} 1\right)>0$.

We have $(*)$ as follows: Let $\xi \in \cap_{i=0}^{\infty} B_{i}$. Let $B(x):=\{n \mid$ $10^{n} 1$ appear in $\left.x\right\}$, where we write $10^{0} 1=11$. Since $k_{1}-$ $k_{0} \geq 1$, from (17) and (21), we have $11 \sqsubset \phi(\xi)^{\prime}$. Let $x_{0}:=11$ then $(*)$ trivially holds for $x_{0}$ and $B\left(x_{0}\right)=\{0\}$. From (17) and (21), there are $x_{n-1} \in S$ and $k \geq 1$ such that $x_{n-1} y 1 \sqsubset \phi(\xi)^{\prime}$ for $y:=s\left(\mathcal{C}_{n-1}\right)^{k} 0^{2^{k_{n}-(n+1)}}$. Suppose that $(*)$ holds for $x_{n-1}$ and $B\left(x_{n-1}\right)=\{f(i) \mid 0 \leq i \leq n-1\}$, where $f(0)=0$. Since $B\left(s\left(\mathcal{C}_{n-1}\right)\right) \subseteq B\left(x_{n-1}\right)$ and the first bit of $s\left(\mathcal{C}_{n-1}\right)$ is 1 , we have $B\left(x_{n-1}\right)=B(z)$ for $x_{n-1} \sqsubseteq z \sqsubseteq x_{n-1} y$ and $B\left(x_{n-1} y 1\right)=B\left(x_{n-1}\right) \cup\{f(n)\}$. Let $x_{n}:=x_{n-1} y 1$ then $(*)$ holds for $x_{n}$ and $B\left(x_{n}\right)=\{f(i) \mid 0 \leq i \leq n\}$. By induction, (*) holds for $\phi(\xi)^{\prime}$, see Fig. 3

Let $K:=\left\{\left(i, k_{i}\right) \mid i \in \mathbb{N}\right\}$. Since $10^{n} 1$ and $01^{m} 1$ appear in $\phi(\xi)^{\prime}$ iff $P\left(10^{n} 1\right)>0$ and $P\left(01^{m} 1\right)>0$. From (20) and (*), we can compute $K$ from $\phi(\xi)^{\prime}$ if $\xi \in \cap_{i} B_{i}$. Thus there is a partial computable $g$ such that (i) $g(x)$ is defined then $g(x)=g(z)$ for $x \sqsubseteq z$, (ii) $g(\omega):=\cup_{x \sqsubset \omega} g(x)$ and (iii)

$$
P\{\omega \mid g(\omega)=K\} \geq \lambda\left(\cap_{i=0}^{\infty} B_{i}\right)>0 .
$$




$$
\begin{array}{cccccccccccc}
\mathcal{C}_{n} & =\overbrace{\mathcal{C}_{n-1}^{1} * \cdots}^{B_{n}} * \mathcal{C}_{n-1}^{2^{k_{n}-k_{n-1}}} \\
L_{1} & L_{2} & L_{3} & \cdots & L_{7} & L_{8} & L_{9} & L_{10} & L_{11} & L_{12} & L_{13} & L_{14} \\
1 & 1 & 0 & \cdots & 1 & 1 & 0 & 1 & 1 & 0 & 0 & 0 .
\end{array}
$$

Fig. 3. $\mathcal{C}_{n-1}^{i}, 1 \leq i \leq 2^{k_{n}-k_{n-1}}$ is a $2^{k_{n}-k_{n-1}}$ partition of $\mathcal{C}_{n-1}$. Note that $s\left(\mathcal{C}_{n-1}\right)$ ends with $10^{f(n-1)}$ and does not contain the pattern $10^{f(n-1)} 1$. Since $s\left(\mathcal{C}_{n-1}\right)$ starts with 1 , the trajectories starting from $B_{n}$ contain the pattern $10^{f(n-1)} 1$. For example, let $k_{0}=1, k_{1}=2, k_{2}=$ 4 then $s\left(\mathcal{C}_{0}\right)=1, s\left(\mathcal{C}_{1}\right)=110, s\left(\mathcal{C}_{2}\right)=11011011011000 . B_{0}$ is the union of $L_{i}$ such that $s\left(L_{i}\right)=1 . B_{0} \cap B_{1}=L_{1} \cup L_{4} \cup L_{7} \cup L_{10}$, and $B_{0} \cap B_{1} \cap B_{2}=L_{1} \cup L_{4} \cup L_{7}$. The trajectories starting from $B_{0} \cap B_{1}$ always contain the pattern 11 and those from $B_{0} \cap B_{1} \cap B_{2}$ always contain patterns 11 and 101.

$$
\begin{aligned}
& \text { Let } \\
& \qquad P_{n}(x):=\lambda\left(\cup\left\{L_{i} \mid \exists 1 \leq i \leq j \leq h, x=s\left(L_{i} \ldots L_{j}\right)\right\}\right),
\end{aligned}
$$

for $\mathcal{C}_{n}=\left(L_{1}, \ldots, L_{h}\right)$. We have $P_{n}(x) \leq P_{n+1}(x)$ and $P_{n}(x) \geq P_{n}(x 0)+P_{n}(x 1)$ for all $n$ and $x$. Since (i) $P_{n}(x)$ is computable from $s\left(\mathcal{C}_{n}\right)$ and $w\left(\mathcal{C}_{n}\right)=2^{-k_{n}}$ and (ii) $s\left(\mathcal{C}_{n}\right)$ is computable from $k_{0}, \ldots, k_{n}$, we have that $P_{n}(x)$ is computable from $k_{0}, \ldots, k_{n}$. Since $\lambda\left(\cup_{n} S\left(\mathcal{C}_{n}\right)\right)=1$, we have $\lim _{n} P_{n}(x)=P(x)$. Since $P$ is a probability, we can compute $P(x)$ with any given precision from $K$. Thus $P$ is effectively estimated from $\phi(\xi)^{\prime}, \xi \in \cap_{i} B_{i}$.

Finally we show that the entropy is zero. Since $w\left(\mathcal{C}_{n}\right)=$ $2^{-k_{n}}$ and $h\left(\mathcal{C}_{n}\right)=2^{k_{n}}\left(1-2^{-(n+1)}\right)$, we have

$$
\lim _{n} \frac{-\log _{2} P\left(s\left(\mathcal{C}_{n}\right)\right)}{h\left(\mathcal{C}_{n}\right)}=0
$$

Since $\lambda\left(\cup_{n} S\left(\mathcal{C}_{n}\right)\right)=1$, from a similar argument for the previous theorem, we see that the entropy is zero.

Lemma 2. Let $\mathcal{C}:=\left(L_{1}, \ldots, L_{h}\right)$ and $\left(L_{1}^{\prime}, \ldots, L_{2^{k} h}^{\prime}\right):=$ $\mathcal{C}(k)$. Then for $0 \leq n \leq 2^{k}-1$ and $J \subseteq\{1, \ldots, h\}$,

$$
\begin{aligned}
& \cup_{j \in J} L_{j} \cap \cup_{i=n h+1}^{(n+1) h} L_{i}^{\prime}=\cup_{j \in J^{\prime}} L_{j}^{\prime}, J^{\prime}=\{j+n h \mid j \in J\}, \\
& \lambda\left(\cup_{j \in J^{\prime}} L_{j}^{\prime}\right)=2^{-k} \lambda\left(\cup_{j \in J} L_{j}\right) .
\end{aligned}
$$

Proof) Since $\mathcal{C}(k)$ is a concatenation of $2^{k}$ columns of the same width partition of $\mathcal{C}$, the lemma follows.

\section{Acknowledgement}

The author thanks Prof. Teturo Kamae (Matsuyama Univ.), Prof. Benjamin Weiss (Hebrew Univ.), and anonymous referees for helpful discussions and valuable comments.

\section{REFERENCES}

[1] D. H. Bailey. Sequential schemes for classifying and predicting ergodic processes. PhD thesis, Stanford Univ., 1976.

[2] T. M. Cover. Open problems in information theory. In 1975 IEEE Joint Workshop on Information Theory, pages 35-36, 1975.

[3] K. de Leeuw, E. F. Moore, C. E. Shannon, and N. Shapiro. Computability by probabilistic machines. In C. E. Shannon and J. McCarthy, editors, Automata Studies, pages 183-212. Princeton Univ. Press, 1956.

[4] L. Györfi, G. Morvai, and S. J. Yakowitz. Limits to consistent online forcasting for ergodic time series. IEEE Trans. Inform. Theory, 44(2):886-892, 1988.

[5] D. S. Ornstein. Guessing the next output of a stationary process. Israel J. Math., 30(3):292-296, 1978.
[6] B. Ya. Ryabko. Prediction of random sequences and universal coding. Probl. Inf. Transm., 24:87-96, 1988.

[7] P. Shields. The ergodic theory of discrete sample paths. Amer. Math. Soc., 1996.

[8] V. V. V'yugin. Ergodic theorems for individual random sequences. Theor. Comp. Sci., 207:343-361, 1998.

[9] B. Weiss. Single Orbit Dynamics. Amer. Math. Soc., 2000. 